\title{
Um Estudo da Cadeia de Valores com a Utilização da Análise Fatorial
}

\author{
Roberto Giro Moori \\ Moisés Ari Zilber
}

\begin{abstract}
Resumo
O objetivo deste estudo foi avaliar a importância das variáveis que compõem a cadeia de valores como fatores contribuintes à vantagem competitiva das empresas e sua relação com a gestão da cadeia de suprimentos. Nesse sentido foi realizada pesquisa de campo junto a destacados dirigentes de empresas, que direta ou indiretamente interagem com os aspectos relativos às variáveis consideradas. Essas foram definidas como as que compõem a escala de valores de Porter (1985). Os dados foram obtidos a partir de uma amostra por conveniência, composta por 62 empresas, analisadas por meio de estatística descritiva e multivariada, especificamente pela técnica da análise fatorial. Com base nessa amostra, os resultados evidenciaram que houve deslocamento de algumas variáveis, agrupadas por Porter (1985) como pertencentes às atividades primárias, para as atividades de apoio e vice-versa. Além disso, foram identificados dois fatores ou componentes principais, denominados gestão da administração e logística e operações, indicando que a cadeia de suprimentos tem significativa importância nesse processo, com a ressalva de que as variáveis desenvolvimento de tecnologia e aquisição contribuiram em menor intensidade do que as demais variáveis.
\end{abstract}

Palavras-chaves: competitividade; cadeia de suprimentos; capacitação gerencial; cadeia de valores; vantagem competitiva.

\section{Abstract}

The objective of this study was to evaluate the importance that companies give to all variables that create value chains as contributors to the competitive advantage of the companies and its relation with supply chain management. In this way a field research was made along with main executives of companies that direct or indirectly interact with aspects related to these variables. These were defined as the ones which create Porter's value scale (1985). Data were obtained from a sample of convenience formed by 100 companies, reaching $62 \%$ feed-back. The statistic analysis was developed based on descriptive statistical and multivariate data analysis, specifically factor analysis technique. The results show that there was a movement of variables grouped accordingly Porter (1985), as part of primary activities towards support tasks. Besides, two factor or component principals were identifies, named strategic management and logistics and operations, which lead to the supposition that supply chain has a significant importance in this process. However, technology development and acquisition have contributed less then the other variables.

Key words: competitivity; supply chain; management capability; value chain; competitive advantage. 


\section{INTRODUÇÃO}

Este trabalho trata da avaliação, mediante a aplicação da análise fatorial, da significância das variáveis que compõem a cadeia de valores de Porter (1985), consideradas as atividades primárias e de apoio, no processo de gestão das empresas para atender os seus objetivos de competitividade no mercado.

Verifica-se, na atualidade, o estabelecimento de nova postura empresarial com reflexos, entre outros, nos sistemas de manufatura, mercados, ciclo de vida dos produtos, estoques, processos produtivos, mão-de-obra, estruturas organizacionais e marketing, em busca da competitividade. A estratégia competitiva, conforme Porter (1998), é a busca de posição competitiva favorável em uma indústria, arena fundamental onde ocorre a concorrência, visando a estabelecer uma posição lucrativa e sustentável contra as forças que determinam a concorrência. Essa determinação empresarial leva à possibilidade de expansão, de diversificação ou até mesmo de sobrevivência de uma empresa, especialmente se a sua ação envolve mercados competitivos. As competências distintivas permitem a criação e a manutenção de vantagem competitiva (Prahalad, Fahey e Randall, 1994; Aaker, 1998; Jennings, 1998). A base do conceito é integrar os processos logísticos e comerciais ao longo de toda a cadeia de abastecimento, buscando sinergia em que o todo é mais benéfico do que a simples soma das partes (Ansoff, 1990). Induz-se a empresa a adquirir novas tecnologias, investir em métodos e processos e estabelecer alianças.

Emergem no processo econômico atual significativas funções relativas às cadeias de suprimentos, que podem revelar diferenciações ou reduções de custos dos produtos, que efetivam a vantagem competitiva (Friedman e Furey, 1999).

A busca da competitividade mediante a prestação de serviços fundamentais ou core business requer a aplicação de novas tecnologias, visando à melhoria dos equipamentos e dos produtos, à redução dos custos e ao aumento da produtividade. Nessas condições, as empresas envidam esforços no sentido de melhoria contínua, porque não podem mais contar, em termos estratégicos, apenas com preços eventualmente menores, mão-de-obra de menor custo ou com o acesso fácil aos recursos naturais como fatores preponderantes para alcançar a competitividade numa escala global.

Dentro de um particular segmento de mercado é a relativa vantagem competitiva que determina a lucratividade e o crescimento de uma empresa, conforme 
argumentam Prahalad e Hamel (1998). Ela está alcançando quase todos os setores econômicos. É uma combinação de desregulamentação, que permite a entrada de novos competidores, com uma regulamentação básica, que reduz o risco de manipulação do mercado por intermediários, tornando possível que ele se desenvolva livremente.

A competitividade em mercado globalizado se dá com a posse ou não de tecnologias produtivas ou de informação, bem como da capacidade de inovação. A força de uma estratégia não é determinada por um movimento inicial, mas muito mais pela antecipação dos movimentos dos competidores e mudanças nas demandas dos consumidores ao longo do tempo, conforme afirma Day (1997).

A estratégia fornece a direção e sentido para que uma companhia defina seu negócio e saiba aonde pode e quer chegar. Não se pode dissociar dela a questão da competitividade. Dentro da concepção de estratégia de uma empresa, certamente reside a consciência de ser competitiva. Nesse sentido ela deverá aplicar seus recursos, especialmente os investimentos, de modo a estar preparada, em termos de tecnologias, métodos e processos que lhe permitam produzir o melhor produto, a preços atraentes aos consumidores e em condições de disponibilidade e acesso instantâneo a ele.

Por trás de uma empresa de sucesso há superior estratégia, afirma Markides (1999). Segundo ele, a companhia pode tê-la desenvolvido por meio de análises formais, tentativa e erro ou por pura sorte; mas alguma estratégia sustentou a rota dessa empresa para o sucesso. Porter (1985) sugere que, para diagnosticar a vantagem competitiva de uma empresa, é necessário definir a sua cadeia de valores. Começando com a cadeia genérica, as atividades de valor são identificadas individualmente.

Considerando essas posições, buscou-se identificar em Porter (1985) quais as atividades estratégicas ligadas à cadeia de valores que, se desenvolvidas pela empresa, implicariam vantagens competitivas para ela. Assim, a pesquisa desenvolvida procurou responder a esta questão: entre as variáveis componentes da escala de valor de Porter (1985), quais delas ascendem como prioritárias dentro das suas estratégias de buscar vantagens competitivas?

Ao procurar responder a esse problema de pesquisa, este trabalho tem estes objetivos: (1) verificar se as variáveis que compõem as atividades primárias e de apoio da cadeia de valor de Porter (1985), se mantêm ou mudam de categoria; e (2) se nesse processo estratégico, as empresas consideravam as variáveis na gestão da cadeia de suprimentos (supply chain management), como fator preponderante para alcançar tanto a vantagem em custo como a vantagem em valor. 


\section{Cadeia de Valores e Gestão da Cadeia de Suprimentos}

Para Porter (1985) toda empresa é uma reunião de atividades executadas para projetar, produzir, comercializar, entregar e sustentar seus produtos. Todas estas atividades podem ser representadas, fazendo-se uso de uma cadeia de valores. A cadeia de valores desagrega uma empresa nas suas atividades de relevância, para que se possa compreender o comportamento dos custos e as fontes existentes e potenciais de diferenciação. Uma empresa ganha vantagem competitiva, executando estas atividades estrategicamente importantes de forma mais eficaz. Essas atividades, segundo o autor, denominadas de atividades de valor, podem ser divididas em dois tipos: atividades primárias e atividades de apoio.

As atividades primárias são aquelas envolvidas na criação física do produto e na sua venda e transferência para o comprador, bem como na assistência após a venda. Em qualquer empresa, as atividades primárias podem ser divididas em cinco categorias genéricas: (1) logística interna - são as atividades associadas ao recebimento, armazenamento e distribuição de insumos no produto, como manuseio de material, armazenagem, controle de estoque, programação de frotas, veículos e devolução para fornecedores; (2) operações - são as atividades associadas à transformação dos insumos no produto final, como trabalho com máquinas, embalagens, montagem, manutenção de equipamentos, testes, impressão e operações de produção; (3) logística externa - são as atividades associadas à coleta, armazenamento e distribuição física do produto para compradores, como armazenagem de produtos acabados, manuseio de materiais, operações de veículos de entrega, processamento de pedidos e programação; (4) marketing e vendas são as atividades associadas a oferta de um meio pelo qual compradores possam comprar o produto e a induzi-los a fazer isto, como propaganda, promoção, força de vendas, cotação, seleção de canal, relações com canais e fixação de preços; e (5) assistência técnica - são atividades associadas ao fornecimento de serviço para intensificar ou manter o valor do produto, como instalação, conserto, treinamento, fornecimento de peças e ajuste do produto.

As atividades de apoio podem ser divididas em quatro categorias genéricas. Da mesma forma que as atividades primárias, cada categoria de atividades de apoio pode ser dividida em uma série de atividades de valor distintas e específicas a uma determinada empresa. As categorias genéricas das atividades de apoio são: (1) aquisição - refere-se à função de compra de insumos empregados na cadeia de valor da empresa, e não aos próprios insumos adquiridos. Insumos adquiridos incluem matérias primas, suprimentos e outros itens de consumo, bem como ativos como máquinas, equipamento de laboratório, equipamento de escritório e prédios. Embora estes insumos adquiridos estejam comumente associados a atividades 
primárias, eles estão presentes em cada atividade de valor, incluindo atividades de apoio; (2) desenvolvimento de tecnologia - consiste em várias atividades que podem ser agrupadas, em termos gerais, em esforços para aperfeiçoar o produto e o processo. $\mathrm{O}$ desenvolvimento de tecnologia relacionada ao produto e às suas características pode apoiar toda a cadeia produtiva; (3) gerência de recursos humanos - consiste em atividades envolvidas no recrutamento, na contratação, no treinamento, no desenvolvimento e na compensação de todos os tipos de pessoal. A gerência de recursos humanos afeta a vantagem competitiva em qualquer empresa, por meio do seu papel na determinação das qualificações e do custo da contratação e do treinamento; e (4) infra-estrutura da empresa - consiste em uma série de atividades, incluindo gerência geral, planejamento, finanças, contabilidade, jurídicas, questões governamentais e gerência de qualidade. A infraestrutura, ao contrário de outras atividades de apoio, geralmente dá suporte a toda a cadeia produtiva e não apenas às atividades individuais.

A cadeia de suprimentos, por outro lado, representa um conjunto de empresas que contribui seqüencialmente para a produção e a distribuição de produtos, que vai desde as atividades extrativas até o consumidor final. Tradicionalmente, a maior parte das organizações entende que as empresas existem independentes umas das outras e, que na realidade, precisam competir entre si para sobreviver. Entretanto tal filosofia pode ser autodestrutiva, se ela conduzir a falta de boa vontade de cooperar, portanto, para competir. Por trás deste conceito, aparentemente paradoxal, está a idéia de integração da cadeia de suprimentos. Por exemplo, um fabricante de carros é parte de uma cadeia que se estende, para trás, para a siderurgia, para o fabricante de auto peças, e, para frente, para os distribuidores e varejistas, até o consumidor final. Cada uma dessas empresas na cadeia é dependente da outra por definição e, ainda paradoxalmente, por tradição elas não cooperam umas com as outras.

Atualmente, as organizações estão cada vez mais enfocando seus negócios nas atividades que elas fazem realmente bem e nas quais possuem vantagem diferencial. O restante é “adquirido externamente”, isto é, obtido fora da empresa, conforme argumenta Christopher (1992).

Com a competição, agora em nível mundial, o excesso de oferta de produtos, a demanda reprimida e os avanços das tecnologias de informação e comunicação fazem com que a gestão da cadeia de suprimentos possa significar aumento da qualidade do produto e redução de custos e preços, acompanhados pela sua maior e melhor disponibilidade ao consumidor.

A cadeia de suprimentos engloba todas as atividades associadas com o fluxo e transformação de bens, desde as matérias primas até o consumidor final e os sistemas de informações. Handfield e Nichols (1999) defendem que cadeia de 
suprimento é a integração dessas atividades por meio do desenvolvimento de relações para adquirir vantagens competitivas.

Assim, é importante combinar vantagens competitivas com outras empresas na busca de alianças estratégicas e de redes interempresas. Investimentos em sistemas de comunicação, sistemas operacionais de computadores e acesso em alta velocidade à Internet podem facilitar a integração interempresas, mesmo em nível mundial. A celebração de alianças com outras empresas permite, por exemplo, a aquisição de produtos ou processos tecnológicos, a repartição dos custos, a diluição dos riscos, em especial de pesquisas e desenvolvimento, acesso a novos canais e mercados de distribuição.

\section{Oportunidades na Cadeia de Suprimentos}

Acredita-se que a proposta de investir capital na própria cadeia de suprimentos na qual o investidor possui negócios, possa constituir aspecto fortemente atrativo, visando ao fortalecimento da referida cadeia e, conseqüentemente, da rentabilidade e/ou valor agregado do negócio em que o investidor opera. Por outro lado, este enfoque permite visualizar a cadeia de suprimentos como condomínio integrado de empresas, em relação de parceria: além do atendimento dos interesses próprios de cada uma, todas se constituem numa entidade única. Como conjunto, elas investem em interesses comuns, gerando até mesmo oportunidades de novos negócios. As capacidades da organização, tais como os conhecimentos adquiridos, têm importante papel nas suas opções estratégicas inovadoras (Rea e Kerzner, 1997; Grant, 1998). Sem estratégia de inovação, não é possível identificar uma direção a seguir (Cooper, 2000). A estratégia de desenvolvimento de novos produtos é elaborada no nível funcional da organização (Aaker, 1998). Conforme Copacino (1997), para os executivos o principal objetivo é maximizar o valor do acionista. Nesse sentido torna-se cada vez mais sofisticado analisar e medir esse retorno. Uma das abordagens, segundo ele, é baseada na relação: Criação de valor para o acionista $=$ Caixa - (Capital Investido $\mathrm{x}$ Custo do Capital), sendo Caixa medido pelo lucro operacional depois dos impostos. Ou seja, qualquer ação na direção de diminuir custos de logística expande o Caixa, o que, mantidas fixas as demais variáveis, cria valor para o acionista. A melhor gestão de estoques, por exemplo, deve implicar redução de custos, conforme Anvari (1992), o que determinará aumento de rentabilidade para o acionista.

Robles (1998) considera que os investimentos em logística propiciam a diminuição de custos totais com a distribuição, reduzindo os estoques, o tempo de ressuprimento, o processamento de pedidos (levantamento, registros, controle, 
expedição), os custos de transporte, de manuseio, entre outros, facilitando até mesmo a coordenação de planejamento e de gerenciamento entre fornecedores e distribuidores, que ao final garantirá melhor atendimento ao mercado.

A integração da cadeia de suprimentos está modificando a forma de se fazer negócios. Esta compreensão tem estimulado os administradores a oferecer seus produtos ou serviços aos seus clientes de maneira mais rápida, mais barata e melhor que a concorrência. Assim, para ganhar competitividade, está claro que isso não poderá ser conseguido isoladamente, mas de modo cooperativo com outras organizações.

Narus e Anderson (1996) argumentam que não importa quão amplo seja o rol de serviços disponível. Quando um consumidor necessita de alguns serviços, além dos habituais que o fornecedor oferece, independentemente de quanto esforço ele dispenda para atendê-lo, o que ocorre na maior parte das vezes é insuficiência de condições para satisfazer a urgência do consumidor. Em casos como esse, a eventual existência de um sistema adequado de suprimentos permitiria atendimento com maior presteza.

A idéia de cadeia de suprimentos está relacionada com os aspectos estratégicos da empresa, uma vez que envolve variáveis estratégicas como mercado, receitas, custos e lucros. Para Li e O’Brien (1999), a fim de aprimorar a eficiência e a eficácia do canal de suprimento, deve-se focar quatro critérios: lucro, tempo de resposta, rapidez de entrega e eliminação de resíduos.

A eficiência e a eficácia da organização devem ser pensadas e dirigidas para o atendimento de seus clientes. Atitudes como selecionar oportunidades, atender clientes, criando e fornecendo valor para eles, são representativas e significantes (Pascale, 1996; Ohmae, 1998). Esses autores defendem explicitamente a criação de valor, o atendimento das reais necessidades dos clientes e a promoção da sua satisfação. Conforme Zairi (1997), as estratégias vencedoras estão fundamentadas numa combinação de critérios que enfatizam cada vez mais o mercado e o consumidor final, em lugar de operações internas.

A importância da cadeia de suprimentos em relação à estratégia de competitividade de uma empresa com o objetivo de criar valor a seus clientes, deve compatibilizar habilidades e recursos com as oportunidades encontradas no meio ambiente externo a ela, minimizando custos de operação ou diferenciado-a da concorrência. De acordo com Porter (1998), a vantagem competitiva surge fundamentalmente do valor que uma empresa consegue criar para os seus compradores, que ultrapassa o custo de fabricação e a base do desempenho acima da média a longo prazo só pode ser conseguida a partir de baixo custo ou diferenciação. A utilização da cadeia de suprimentos é promissora, porque, sendo 
o mercado o conjunto dos clientes atuais e potenciais da empresa e também os da concorrência, em situação de ambiente cada vez mais competitivo, haverá maior vantagem para aquelas que adotarem e implementarem sistemas de cadeia de suprimentos, que podem ser concretizados na forma de investimentos nas linhas de produção, distribuição ou em outras funções operacionais

\section{Metodologia}

\section{Amostra e Coleta dos Dados}

O método da pesquisa foi de natureza exploratória do tipo descritivo, tendo-se adotado amostra não probabilística. Por isso as inferências extraídas da amostra devem ser ressalvadas, em função das limitações deste tipo de abordagem (Kerlinger, 1980). Assim, uma vez definido o perfil das empresas como localidade, número de funcionários, tipo de produto ou serviços prestados e outros, foram escolhidas por conveniência 100 empresas para a coleta dos dados. O instrumento utilizado foi um questionário composto de questões fechadas, enviado aos executivos das áreas de planejamento, controle e suporte às atividades primárias ou de apoio à empresa, isto é, envolvidos direta ou indiretamente com o tema deste estudo. O levantamento de dados ocorreu no primeiro trimestre de 2000.

O questionário foi estruturado em dois blocos:

- no primeiro bloco procurou-se levantar sucintamente o perfil dos respondentes e da empresa;

. o segundo bloco tratou da coleta dos dados relacionados às atividades primárias e de apoio da cadeia de valor. A definição das atividades foi feita com base nos conceitos emitidos por Porter (1985). Assim, este bloco foi estruturado com nove assertivas, referentes aos dois aspectos das atividades de valor, sendo: (1) cinco atividades primárias, relacionadas à logística de entrada, operações, marketing e vendas, logística de saída e assistência técnica após a venda; e (2) quatro atividades de apoio, relacionadas à infra-estrutura da companhia, gerenciamento dos recursos humanos, desenvolvimento de tecnologia e aquisição.

No questionário, as assertivas apareceram sem a identificação do grupo ou categoria a que pertenciam, para evitar tendências nas respostas. Para tanto, foi utilizada uma escala de Discordância/ Concordância, composta de cinco pontos, do tipo Likert, em que valores menores indicavam maior concordância com relação 
à afirmação apresentada e os valores maiores indicavam maior discordância com a afirmação relacionada aos aspectos relevantes de vantagem competitiva, estabelecidos por Porter (1985).

\section{Tratamento dos Dados}

Para o tratamento dos dados coletados, utilizou-se a estatística descritiva e a técnica da análise fatorial. Na aplicação destas estatísticas foram tomados alguns cuidados, conforme se mostra a seguir.

\section{Estatística Descritiva}

A estatística descritiva foi utilizada com a finalidade de resumir algumas características da amostra. O tipo de variável utilizado para a coleta dos dados foi o qualitativo em escala ordinal. Assim, utilizou-se a freqüência e a moda como um indicador de média, como é usual quando se trata com dados ordinais, cuja distribuição é não-paramétrica. Observa-se que, muitas vezes, é tentador ir além, tratando os dados ordinais como se fossem de intervalos e usar estatísticas paramétricas, embora ressalte Easterby-Smith (1999) que a rigor é incorreto, mas com amostra grande e dimensão contínua como Discordo Totalmente até Concordo Totalmente, o resultado comumente é preciso e útil.

\section{Análise Multivariada - Técnica da Análise Fatorial}

A análise fatorial (factor analysis) é análise multivariada que se aplica à busca de identificação de fatores em conjunto de medidas realizadas. Basicamente, a análise fatorial pode ser usada nos seguintes casos: (1) para descobrir a estrutura de um conjunto de medições; (2) para reduzir a grande massa de dados da pesquisa em quantidade gerenciável; (3) para auxiliar o pesquisador na construção de escalas, reagrupando as variáveis em fatores independentes e atribuindo o peso a ser usado em cada variável, para ser combinado na escala; e (4) para identificar fatores não correlacionados. Evidentemente, argumenta Pereira (1999), em algumas ocasiões é possível intuir que várias medidas podem compor um fator (ou indicador). No entanto é preferível, ao invés de propor subjetivamente a criação de um indicador, submeter os dados da amostra a uma análise fatorial que aponte objetivamente para essa agregação de medidas.

A condução da análise fatorial exige três passos, conforme cita Mattar (1998): (1) Cálculo das correlações entre as variáveis. As variáveis devem ser correlacionadas duas a duas; (2) Extração inicial de fatores. O objetivo da extração de fatores é encontrar um conjunto de fatores que formem uma combinação 
linear das variáveis na matriz de correlações; (3) Rotação da matriz. A rotação de uma matriz nada mais é que um artifício para prover maior distinção das relações encontradas.

Na aplicação da análise fatorial devem-se considerar e examinar algumas premissas sobre a natureza dos dados. A primeira diz respeito à natureza métrica das medidas, sugerindo que o pesquisador analise a distribuição de freqüência de suas variáveis. Para isso pode-se utilizar o Kaiser-Meyer-Olkin Mesaure of Sampling Adequacy (KMO). O KMO próximo a 1 indica perfeita adequação dos dados para a análise fatorial. Outro teste que pode ser aplicado à análise fatorial é o Cronbach's Alpha, que mede a consistência ou confiabilidade do fator gerado em torno das suas variáveis. O resultado é satisfatório para um valor próximo a 1.

\section{Análise dos Dados}

O objetivo deste ítem é analisar os dados resultantes das tabulações estatísticas dos dados obtidos na pesquisa de campo. As considerações analíticas estão estruturadas em duas partes. Na primeira são analisados os dados de caracterização da amostra e na segunda parte são analisados os aspectos das atividades primárias e atividades de apoio.

\section{Estatística Descritiva}

Visando a contribuir na interpretação dos resultados e subsidiar as inferências, procedeu-se à caracterização dos respondentes, incluindo dois grupos: setor econômico das empresas e funções dos respondentes, conforme mostrados nas Tabelas 1 e 2, respectivamente.

Tabela 1: Setor Econômico das Empresas

\begin{tabular}{l|c|c}
\hline \multicolumn{1}{c|}{ Setor } & Número de empresas & \% \\
\hline Industrial & 36 & 58 \\
Comercial & 11 & 18 \\
Serviços & 15 & 24 \\
\hline \multicolumn{1}{c|}{ TOTAL } & $\mathbf{6 2}$ & $\mathbf{1 0 0}$
\end{tabular}

Constatou-se ter sido expressivo o número de respondentes que pertenciam ao setor industrial, como se pode verificar na tabela acima. 


\section{Tabela 2: Funções dos Respondentes}

\begin{tabular}{l|c|c}
\hline \multicolumn{1}{c|}{ Função } & Número de respondentes & \% \\
\hline Sócio, diretor & 20 & 32 \\
Gerentes & 15 & 24 \\
Supervisores, coordenadores & 19 & 31 \\
Outros TOTAL & 8 & 13 \\
\hline \multicolumn{1}{c|}{ TOT } & $\mathbf{1 0 0}$
\end{tabular}

Em relação às funções desempenhadas pelos respondentes, a Tabela 2 mostra que 56\% da amostra correspondeu à alta administração representada por sócios, diretores e gerentes.

A estatística descritiva foi utilizada para proceder à uma primeira análise e à interpretação das variáveis que influenciaram a vantagem competitiva. Os resultados estão na Tabela 3.

Tabela 3: Fatores de Influência na Vantagem Competitiva

\begin{tabular}{c|c|c|c|c|c|c}
\hline \multirow{2}{*}{ VARIÁVEIS } & \multicolumn{3}{|c|}{ ESCALA } & \multirow{2}{*}{ Moda } \\
\cline { 2 - 6 } & CP & C & I & D & DP & \\
\hline Infra-estrutura da empresa & 20 & $\mathbf{2 7}$ & 8 & 4 & 3 & C \\
Logística de entrada & $\mathbf{3 3}$ & 27 & 1 & 1 & 0 & $\mathrm{CP}$ \\
Gerenciamento dos recursos humanos & 12 & $\mathbf{2 2}$ & 15 & 6 & 7 & $\mathrm{C}$ \\
Marketing e vendas & $\mathbf{1 9}$ & $\mathbf{1 9}$ & 17 & 4 & 3 & $\mathrm{CP} \mathrm{e} \mathrm{C}$ \\
Desenvolvimento de tecnologia & 12 & $\mathbf{2 7}$ & 14 & 7 & 2 & $\mathrm{C}$ \\
Logística de saída & $\mathbf{3 3}$ & 20 & 8 & 1 & 0 & $\mathrm{CP}$ \\
Aquisição & 23 & $\mathbf{2 7}$ & 7 & 4 & 1 & $\mathrm{C}$ \\
Operações & 15 & $\mathbf{3 1}$ & 8 & 6 & 2 & $\mathrm{C}$ \\
Assistência técnica & 17 & $\mathbf{2 3}$ & 16 & 5 & 1 & $\mathrm{C}$ \\
\hline
\end{tabular}

Nota: CP = Concordo Plenamente; $\mathrm{C}=$ Concordo; I = Insuficiente (Indeciso); D = Discordo; $\mathrm{DP}=$ Discordo Plenamente.

A Tabela 3 mostra que, entre os fatores relacionados no instrumento de coleta de dados, os níveis de concordância mais elevados corresponderam aos aspectos logística de entrada e logística de saída, com moda na escala Concordo Plenamente. Estes dados acentuam, assim, a importância da gestão da logística e da cadeia de suprimentos. Este resultado foi significativo, pois teve como base opiniões de um grupo de respondentes situados em funções executivas das empresas.

Entre as dificuldades na busca da vantagem competitiva destacaram-se as demais variáveis, como a infra-estrutura da empresa, o gerenciamento dos recursos humanos, o desenvolvimento da tecnologia, a aquisição, as operações e a 
assistência técnica, com moda na escala Concordo. A variável marketing e vendas, embora tenha modas iguais para a região de concordância Concordo Totalmente e Concordo, tem também alta freqüência para a escala Indeciso. Estes resultados, excetuando-se às variáveis da logística, apontam as dificuldades de ajustamento das empresas às variáveis que compõem a cadeia de valores, como fatores contribuintes à vantagem competitiva.

\section{Técnica da Análise Fatorial}

Efetuada a análise dos dados em termos descritivos, procurou-se, mediante a técnica de análise fatorial, examinar se existiriam dimensões subjacentes às variáveis que pudessem sintetizar, resumir as informações nelas contidas, dando à análise ou à interpretação dos dados da amostra uma melhor compreensão.

O primeiro passo para a condução da análise fatorial foi o cálculo das correlações entre as variáveis, como pode ser visualizado na Tabela 4.

\section{Tabela 4: Matriz de Correlações}

\begin{tabular}{|c|c|c|c|c|c|c|c|c|c|}
\hline VARIÁVEIS & 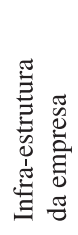 & 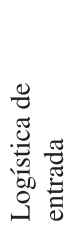 & 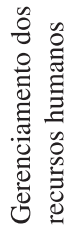 & 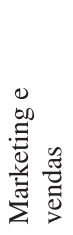 & 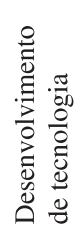 & 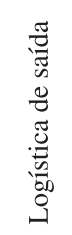 & 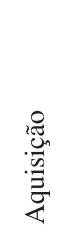 & 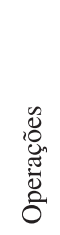 & 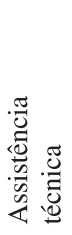 \\
\hline $\begin{array}{l}\text { Infra-estrutura da empresa } \\
\text { Logística de entrada }\end{array}$ & 1 & 1 & & & & & & & \\
\hline Gerenciamento rec. humanos & 0,48 & $-0,35$ & 1 & & & & & & \\
\hline Marketing e vendas & 0,33 & 0,27 & 0,40 & 1 & & & & & \\
\hline Desenvolvimento de tecnologia & 0,35 & 0,07 & 0,53 & 0,31 & 1 & & & & \\
\hline Logística de saída & $-0,06$ & 0,58 & $-0,41$ & 0,11 & $-0,04$ & 1 & & & \\
\hline Aquisição & 0,02 & 0,21 & $-0,14$ & 0,21 & $-0,11$ & 0,36 & 1 & & \\
\hline Operações & 0,24 & 0,35 & $-0,20$ & 0,12 & $-0,01$ & 0,52 & 0,30 & 1 & \\
\hline Assistência técnica & 0,42 & $-0,03$ & 0,38 & 0,46 & 0,37 & $-0,03$ & $-0,02$ & 0,18 & 1 \\
\hline
\end{tabular}

Nota: Kaiser-Meyer-Olkin Measure of Sampling Adequacy (KMO): 0,642; Cronbach's Alpha: 0,655 .

Os resultados sugerem que os dados para essa análise fatorial têm ajuste de adequação aos dados $(\mathrm{KMO}=0,642)$ de $64 \%$ de uma escala de 0 a $100 \%$. Numa perspectiva de análise de confiabilidade (Cronbach's Alpha = 0,655), indicam um nível de $65 \%$, ou seja, suas medidas concordariam em $65 \%$ das vezes. Prosseguindo na análise, nota-se que os maiores coeficientes de correlação estavam entre as variáveis logística de saída $(0,58)$, desenvolvimento de tecnologia $(0,53)$ e operações $(0,52)$. 
Realizado o cálculo das correlações entre as variáveis, o passo seguinte foi a extração dos fatores da matriz de correlação, com o objetivo de encontrar um conjunto de fatores que formassem uma combinação linear das variáveis da matriz de correlação. Desta forma, se as variáveis fossem altamente correlacionadas entre si, elas seriam combinadas para formar um fator, e assim sucessivamente com as demais variáveis da matriz de correlação. Na Tabela 5, têm-se a extração dos componentes principais, constituídos de nove fatores que correspondem às variáveis originais.

Tabela 5: Extração dos Componentes Principais

\begin{tabular}{c|c|c|c|c}
\hline Value & Eingenvalue & $\begin{array}{c}\text { \% total } \\
\text { variance }\end{array}$ & $\begin{array}{c}\text { Cumulative } \\
\text { Eingenvalue }\end{array}$ & $\begin{array}{c}\text { Cumulative } \\
\%\end{array}$ \\
\hline 1 & 2,6672 & 29,6355 & 2,6672 & 29,6355 \\
2 & 2,4057 & 26,7295 & 5,0729 & 56,3650 \\
3 & 0,9042 & 10,0446 & 5,9770 & 66,4113 \\
4 & 0,8493 & 9,4363 & 6,8263 & 75,8476 \\
5 & 0,6708 & 7,4536 & 7,4971 & 83,3011 \\
6 & 0,5657 & 6,3073 & 8,0648 & 89,6084 \\
7 & 0,3978 & 4,4203 & 8,4626 & 94,0287 \\
8 & 0,3331 & 3,7022 & 8,7957 & 97,7302 \\
9 & 0,2043 & 2,2710 & 9,0000 & 100,0000 \\
\hline
\end{tabular}

Examinando-se os eigenvalues, verifica-se que há dois fatores com valores superiores a 1. Esses dois primeiros fatores possuem eigenvalues que correspondem a $29,63 \%$ e $26,72 \%$ do total de eigenvalues do modelo, ou seja, explicariam em conjunto 56,36\% das variâncias das medidas originais.

Existem alguns critérios para a adoção de número de fatores para a construção do modelo fatorial. Entre eles têm-se o critério de Kaiser (Hair et al., 1998) e o teste de scree, proposto por Cattell (apud Hair et al., 1998). O critério de Kaiser, provavelmente o mais usado, estabelece que somente os fatores que possuem eigenvalue maior do que 1 devem permanecer no modelo final. Portanto, na presente pesquisa, permaneceriam somente dois fatores.

O teste scree é um método gráfico em que o número de fatores de carga é determinado pelo aparecimento da inclinação suave no gráfico. Esta inclinação indica a presença de fatores pouco significativos. A Figura 1 mostra a aplicação do teste scree, evidenciando dois fatores de carga, correspondente ao eigenvalue maior do que 1 . 


\section{Figura 1: Teste Scree - Número de Fatores de Carga}

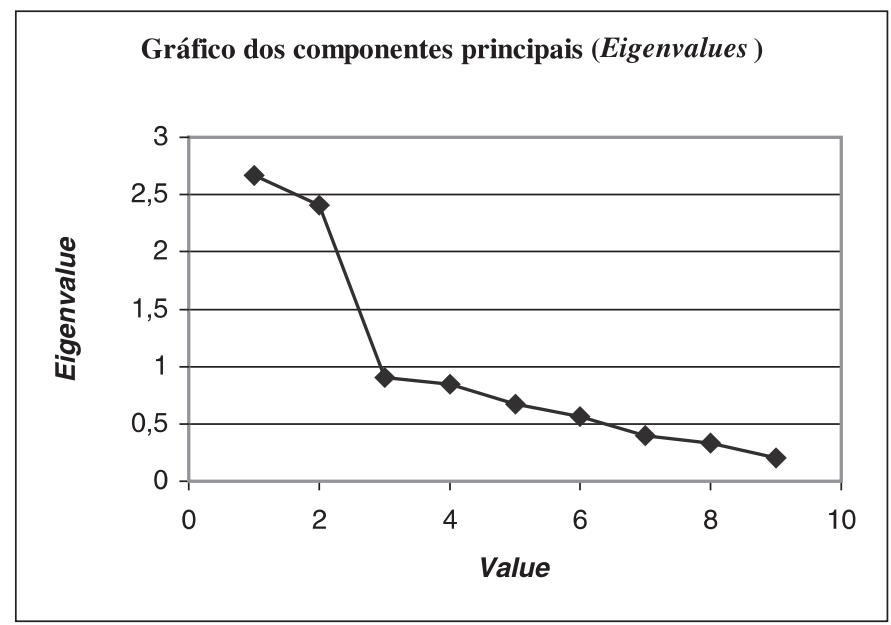

Tomada a decisão sobre critérios para o número de fatores, isto é, cargas das variáveis associadas aos dois fatores mais significativos (de maiores eigenvalues), passou-se ao exame do modelo final, representado na Tabela 6.

\section{Tabela 6: Fator de Carga (Sem Rotação)}

\begin{tabular}{l|c|c|c}
\hline \multicolumn{1}{c|}{ VARIÁVEIS } & Fator 1 & Fator 2 & Communality \\
\hline Infra-estrutura da empresa & 0,6567 & 0,3118 & 0,5285 \\
Logística de entrada & $-0,2679$ & $\mathbf{0 , 7 0 8 7}$ & 0,5741 \\
Gerenciamento dos recursos humanos & $\mathbf{0 , 8 7 1 2}$ & $-0,1497$ & 0,7814 \\
Marketing e vendas & 0,5453 & 0,5089 & 0,5564 \\
Desenvolvimento de tecnologia & 0,6740 & 0,1824 & 0,4875 \\
Logística de saída & $-0,4029$ & $\mathbf{0 , 7 5 5 4}$ & 0,7330 \\
Aquisição & $-0,1913$ & 0,5362 & 0,3242 \\
Operações & $-0,1226$ & $\mathbf{0 , 7 2 9 1}$ & 0,5466 \\
Assistência técnica & 0,6630 & 0,3190 & 0,5413 \\
\hline \multicolumn{2}{c}{ Explicação da variância } & 0,2964 & 0,4057 \\
& \multicolumn{2}{c}{}
\end{tabular}

Constata-se, então, que o primeiro fator subjacente ao conjunto de variáveis foi significativamente representado por uma variável original, o gerenciamento dos recursos humanos $(0,8712)$. Analisando-se o resultado, pode-se concluir que esse fator se referiu às atividades de apoio. $\mathrm{O}$ segundo fator subjacente foi explicado fundamentalmente por três variáveis originais: logística de saída $(0,7554)$, operações $(0,7291)$ e logística de entrada $(0,7087)$. De certa forma, tais resultados sugerem que as atividades primárias poderiam constituir-se em determinantes da vantagem competitiva das empresas pesquisadas. 
O passo final da análise fatorial foi verificar se os fatores, que são dimensões abstratas, poderiam ser interpretados de forma coerente com a natureza dos fenômenos estudados. Para isso, analisou-se a matriz fatorial para se identificar, pelos fatores de carga, quais as variáveis que melhor se correlacionariam com cada fator. Como a derivação dos fatores pela análise de componentes principais se dá por uma sucessão de rotações de eixos, que melhor expresse a dispersão dos dados, no modelo fatorial final as variações das medidas foram maximizadas e as relações entre as dimensões suavizadas. Por isso deve-se preferir buscar informações sobre as relações entre os fatores e as variáveis originais em matriz fatorial rodada.

Há diferentes estratégias de rotação para a matriz fatorial, sendo aqui considerada a rotação ortogonal, que buscou minimizar o número de variáveis com altas cargas num fator (varimax normalizada). A Tabela 7 apresenta a matriz fatorial após a rotação da matriz.

\section{Tabela 7: Fator de Carga (com Rotação - Varimax Normalizada)}

\begin{tabular}{l|c|c|c}
\hline \multicolumn{1}{c|}{ VARIÁVEIS } & Fator 1 & Fator 2 & Communality \\
\hline Infra-estrutura da empresa & $\mathbf{0 , 7 2 4 9}$ & 0,0550 & 0,5285 \\
Logística de entrada & 0,0045 & $\mathbf{0 , 7 5 7 7}$ & 0,5741 \\
Gerenciamento dos recursos humanos & $\mathbf{0 , 7 5 9 3}$ & $-0,4526$ & 0,7813 \\
Marketing e vendas & $\mathbf{0 , 6 9 1 7}$ & 0,2790 & 0,5563 \\
Desenvolvimento de tecnologia & $\mathbf{0 , 6 9 4 5}$ & $-0,0719$ & 0,4875 \\
Logística de saída & $-0,1047$ & $\mathbf{0 , 8 4 9 7}$ & 0,7330 \\
Aquisição & 0,0140 & $\mathbf{0 , 5 6 9 2}$ & 0,3242 \\
Operações & 0,1475 & $\mathbf{0 , 7 2 4 5}$ & 0,5466 \\
Assistência técnica & $\mathbf{0 , 7 3 3 3}$ & 0,0595 & 0,5412 \\
\hline \multicolumn{2}{c}{ Explicação da variância } & 2,6334 & 2,4394 \\
\cline { 2 - 3 } & 0,2926 & 0,2710 \\
\end{tabular}

Os resultados obtidos mediante a aplicação da técnica de análise fatorial, pelo critério varimax normalizada, conforme se observa pela Tabela 7, evidenciou, no primeiro fator, as variáveis infra-estrutura da empresa $(0,7249)$, gerenciamento dos recursos humanos $(0,7593)$, marketing e vendas $(0,6917)$, desenvolvimento de tecnologia $(0,6945)$ e assistência técnica $(0,7333)$. A natureza destas variáveis levaria a inferir que o primeiro fator se referiria principalmente às atividades de apoio, que podem ser denominadas de gestão da administração. O segundo fator evidenciou as variáveis logística de entrada $(0,7577)$, logística de saída $(0,8497)$, aquisição $(0,5692)$ e operações $(0,7244)$. Estas variáveis estariam associadas às atividades primárias, definidas por Porter (1985), cujo fator poderia ser denominado de gestão de logística e de operações. 
Por meio do gráfico mostrado na Figura 2, é possível verificar a localização das variáveis em um sistema de coordenadas criado pelos fatores.

\section{Figura 2: Relações entre os Fatores e Variáveis num Espaço de Fator de Carga}

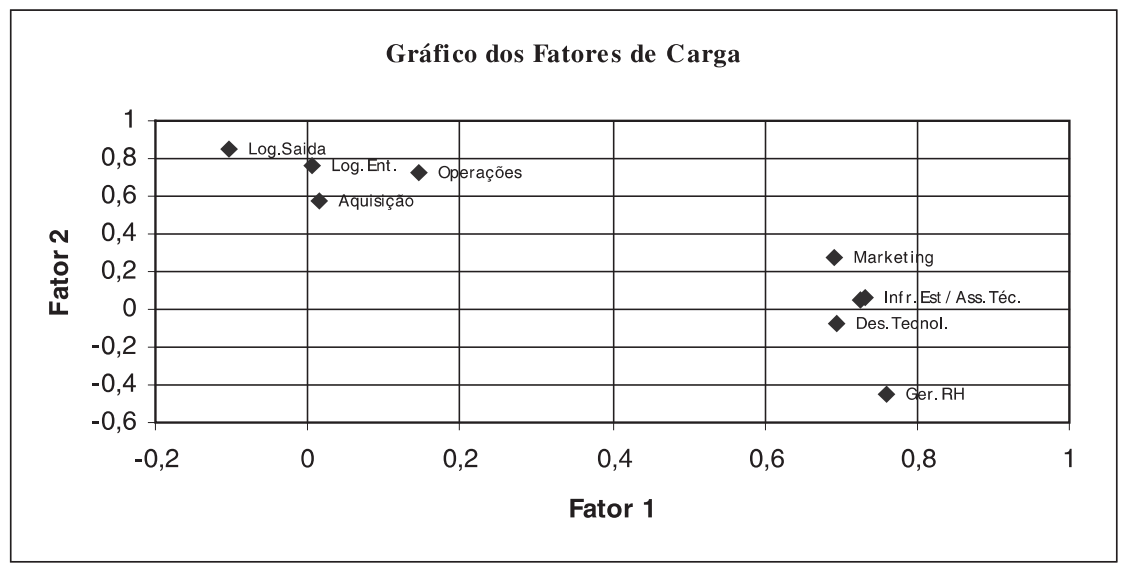

No gráfico fica evidente como as variáveis se agruparam e como ficaram suas relações com os eixos, os factor loadings dos Fatores 1 e 2.

\section{Análise dos Resultados}

Em relação ao objetivo de verificar se as variáveis que compõem as atividades primárias e de apoio da cadeia de valor de Porter (1985), se mantêm ou mudam de categoria, ficou evidenciado que as variáveis componentes das atividades primárias e as de apoio sofreram algumas alterações. As variáveis marketing e vendas e assistência técnica, que pertenciam às atividades primárias, com a aplicação da análise fatorial passaram a pertencer ao grupo das atividades de apoio ou gestão da administração. A variável aquisição, que pertencia à atividade de apoio, passou a pertencer à atividade primária ou gestão da logística e operações.

Além disso, a pesquisa mostrou que as atividades primárias, constituídas das categorias genéricas logística de entrada, operações, logística de saída, marketing e vendas e assistência técnica, e as atividades de apoio, constituídas das atividades genéricas infra-estrutura, gerência dos recursos humanos, desenvolvimento de tecnologia e aquisição, não estão sendo ignoradas pelas empresas. Isto pode ser 
observado pelas correlações significativas, todas acima de 0,50 , embora não haja consenso, de forma geral, entre os pesquisadores, sobre o que seja um bom grau de correlação. Colocando as categorias genéricas, sugeridas por Porter (1985), em confronto com os resultados da pesquisa, obtém-se o exposto na Tabela 8, a seguir.

\section{Tabela 8: Fatores de Carga - Matriz sem Rotação x Matriz com Rotação}

\begin{tabular}{|c|c|c|c|c|c|}
\hline \multirow[t]{2}{*}{ VARIÁVEIS } & \multicolumn{2}{|c|}{$\begin{array}{l}\text { Matriz principal } \\
\text { (sem rotação) }\end{array}$} & \multirow[t]{2}{*}{$\begin{array}{c}\text { Communa- } \\
\text { lity }\end{array}$} & \multicolumn{2}{|c|}{$\begin{array}{c}\text { Matriz com rotação } \\
\text { Varimax normalizada }\end{array}$} \\
\hline & Fator 1 & Fator 2 & & Fator 1 & Fator 2 \\
\hline \multicolumn{6}{|l|}{ Atividades primárias } \\
\hline Logística de entrada & $-0,2679$ & 0,7087 & 0,5741 & 0,0045 & 0,7577 \\
\hline Operações & $-0,1226$ & 0,7291 & 0,5466 & 0,1475 & 0,7245 \\
\hline Logística de saída & $-0,4029$ & 0,7554 & 0,7330 & $-0,1047$ & 0,8497 \\
\hline Marketing e vendas & 0,5453 & 0,5089 & 0,5563 & 0,6917 & 0,2790 \\
\hline Assistência técnica & 0,6630 & 0,3190 & 0,5412 & 0,7333 & 0,0595 \\
\hline \multicolumn{6}{|l|}{ Atividades de apoio } \\
\hline Infra-estrutura da empresa & 0,6567 & 0,3118 & 0,5285 & 0,7249 & 0,0550 \\
\hline Gerenciamento recursos humanos & 0,8712 & $-0,1497$ & 0,7813 & 0,7593 & $-0,4526$ \\
\hline Desenvolvimento de tecnologia & 0,6740 & 0,1824 & 0,4875 & 0,6945 & $-0,0719$ \\
\hline Aquisição & $-0,1913$ & 0,5362 & 0,3242 & 0,0140 & 0,5692 \\
\hline Eigenvalues & 2,6672 & 2,4057 & & 2,6335 & 2,4394 \\
\hline Explicação da variância & 0,2964 & 0,2673 & & 0,2926 & 0,2710 \\
\hline
\end{tabular}

Pela Tabela 8, nota-se que as communalities de cada variável permanecem as mesmas antes e depois da rotação da matriz. Isto mostra que o giro dos fatores, quando feito simultaneamente, aponta exatamente os mesmos valores para cada variável dos fatores não girados e que apenas os pesos de cada variável em cada fator estão agora redistribuídos. Já a quantidade de variação explicada por cada fator mudou da matriz principal (sem rotação) para a matriz com rotação. $\mathrm{Na}$ matriz principal, o Fator 2 tem melhores correlações. Na matriz girada, as cargas foram mudadas de tal forma que as quantidades de variações explicadas são agora aproximadamente idênticas. Na matriz girada fica fácil perceber, pelas grandes cargas atribuídas, quais são as variáveis que compreendem cada fator. Assim o Fator 1, denominado gestão da administração, é composto das variáveis marketing e vendas, assistência técnica, infra-estrutura, gerenciamento dos recursos humanos e desenvolvimento da tecnologia. O Fator 2, denominado gestão de logística e operações, é composto das variáveis logística de entrada, operações, logística de saída e aquisição. As denominações dos fatores foram atribuídas de forma arbitrária. No entanto procurou-se uma denominação que representasse o conjunto das variáveis associadas ao fator.

Com relação ao objetivo de verificar se as empresas consideravam as variáveis na gestão da cadeia de suprimentos (supply chain management) como fatores 
preponderantes para alcançar vantagens em custo e em valor, ficou caracterizado pela estatística descritiva, mostrada na Tabela 3 , que as empresas consideraram essa premissa como meio de alcançar a vantagem competitiva. A moda, como medidor da média da distribuição dos dados, sempre se posicionou no lado concordante da escala. No entanto, pela aplicação da análise fatorial as variáveis aquisição e desenvolvimento de tecnologia tiveram valores de communalities iguais a 0,3242 e 0,4875, respectivamente. Interpreta-se que estas variáveis tiveram pouca contribuição nos resultados na formação dos componentes principais. Este fato, segundo Hair et al. (1998), dependendo do julgamento do pesquisador, poderia ser descartado da análise por não ter uma explicação suficiente na sua formação. Assim, essas duas variáveis devem ser consideradas com ressalvas na gestão da cadeia de suprimentos (supply chain management) das empresas pesquisadas, demonstrando a possibilidade de oportunidades para aplicação de recursos em treinamento, capacitação gerencial e na cadeia de suprimentos.

Esses resultados evidenciam o surgimento de novos processos e métodos no campo da Administração, como a gestão da cadeia de suprimentos e a administração de negócios, desenhando nova configuração de variáveis, condizente com o momento atual.

\section{Conclusões e Sugestões}

A idéia inicial deste estudo foi ampliar a base de discussão sobre questões relativas a temas como produtividade e qualidade, bem como identificar oportunidades de treinamento, capacitação gerencial, investimentos na cadeia de suprimentos e sugerir estratégias que visem a minimizar riscos de fracasso na implementação de projetos na busca de vantagens competitivas das empresas.

As questões relativas à produtividade e qualidade, ligadas à categoria genérica de operações, das atividades primárias e as oportunidades de treinamento, capacitação gerencial e investimentos associadas ao desenvolvimento de tecnologia, relacionadas às atividades de apoio, apresentaram desempenho que pode levar a inferir que as empresas pesquisadas têm consciência da necessidade de se posicionar no mercado e de agir, buscando padrões de competitividade, o que, segundo Christopher (1997), somente ocorre se houver coordenação dos fluxos de materiais e informações que vão do mercado até a empresa e suas operações e, posteriormente, para seus fornecedores.

Por fim, algumas notícias recentes, veiculadas em vários órgãos da imprensa, indicam especial importância e preocupação de empresários com a necessidade 
de buscar eficácia no sistema de distribuição de bens e serviços. Nesse sentido, torna-se fundamental o papel da gestão da cadeia de suprimentos como fator diferenciador de uma empresa em busca de agregar valor ao seu cliente, seja ele outra entidade ou o consumidor final. Esta pesquisa, limitada à amostra estudada, pareceu demonstrar que essa preocupação está evidenciada na ação empresarial.

Sugere-se o prosseguimento deste estudo, ampliando-se a amostra e buscandose, ao mesmo tempo, um estudo comparativo entre setores e condições da propriedade da empresa, nacional ou estrangeira. Deve-se, também, introduzir outras questões, procurando-se fatos explicativos da gestão empresarial a partir deste estudo exploratório.

\section{ReferÊnCIAS Bibliográficas}

AAKER, D. A.

Developing business strategies. 5.ed. New York: John Wiley \& Sons, 1998.

ANSOFF, H. I.

A nova estratégia empresarial. São Paulo: Atlas, 1990.

ANVARI, M.

Eletronic data interchange and inventories. In: CHIKAN, A. Current trends in inventory research. Amsterdan: Elsevier Science Publishers, 1992.

CHRISTOPHER, M.

Logística e gerenciamento da cadeia de suprimentos: estratégias para a redução de custos e melhoria dos serviços. São Paulo: Pioneira, 1997.

COOPER, R. G.

Product innovation and technology strategy. Research Technology Management, Jan./Feb. 2000. Disponível em: <http:// proquest.umi.com/pqdweb>.

COPACINO, W. C.

Supply chain management, the basics and beyond. [S.l.]: The St. Lucie Press, 1997.

DAY, G.

Evaluating strategy alternatives. In: FAHEY, L.; RANDALL, R. M. The portable MBA in strategy. New York: John Wiley \& Sons, 1994.

EASTERBY-SMITH, M.;

THORPE, R.;

LOWE, A.

Pesquisa gerencial em administração. São Paulo: Pioneira, 1999. 
FRIEDMAN, L. G.;

FUREY, T. R.

The channel advantage. Oxford:

Butterworth-Heineman, 1999.

GRANT, R. M.

Contemporary strategy analysis. 3.ed. New York: Blackwell, 1998.

HAIR JR, J. F. et al.

Multivariate data analysis. Englewood Cliffs, NJ: Prentice Hall, 1998.

HANDFIELD, R. B.;

NICHOLS JR, E. L.

Introduction to supply chain management. Englewood Cliffs, NJ: Prentice Hall, 1999.

HENDERSON, B. D.;

LI, D.;

O'BRIEN, C.

Integrated decision modeling of supply chain efficiency. International Journal of Production Economics, v. 59, n. 1-3, 1999.

JENNINGS, D.

Strategic guidelines for outsourcing decisions. In: HUSSEY, D. (Ed.). The strategic decision challenge. New York: John Wiley \& Sons, 1997.

KERLINGER, F. N.

Metodologia da pesquisa em ciências sociais: um tratamento conceitual. São Paulo: EPU/ EDUPS, 1980.
MARKIDES, C. C.

In search of strategy. Sloan

Management Review, Spring 1999.

MATTAR, F. N.

Pesquisa de marketing. São Paulo: Atlas, 1988.

MONTGOMERY, C. A.;

PORTER, M. E.

Estratégia: a busca da vantagem competitiva. 4.ed. Rio de Janeiro: Campus, 1998.

NARUS, J. A.;

ANDERSON, J. C.

Rethinking distribution: adaptive channels. Harvard Business

Review, v. 74, n. 4, 1996.

OHMAE, K.

Voltando à estratégia. In: MONTGOMERY, C. A.; PORTER, M. E. Estratégia: a busca da vantagem competitiva. 4.ed. Rio de Janeiro: Campus, 1998.

PASCALE, R. T.

The Honda effect. In: MINTZBERB, H.; QUINN, J. B. Readings in the strategy process. Englewood Cliffs, NJ: Prentice Hall, 1996.

PEREIRA, J. C. R.

Análise de dados qualitativos. São Paulo: EPU/EDUPS, 1999. 
PORTER, M. E.

Vantagem competitiva: criando e sustentando um desempenho superior. Rio de Janeiro: Campus, 1985.

A vantagem competitiva das nações. Rio de Janeiro: Campus, 1998.

PRAHALAD, C. K.;

FAHEY, L.;

RANDALL, R. M.

A strategy for growth: the role of core competencies in the corporations. In: FAHEY, L.; RANDALL, R. M. (Eds.). The portable MBA in strategy. New York: John Wiley \& Sons, 1994.

PRAHALAD, C. K;

HAMEL, G.

A competência essencial da corporação. Rio de Janeiro: Campus, 1998.

REA, P.;

KERZNER, H.

Strategic planning. New York: VNR, 1997.

ROBLES, L. T.

$O$ caráter estratégico da prestação de serviços de logística integrada na indústria automobilística no Brasil. São Paulo, 1998. Exame de Qualificação (Doutorado) Faculdade de Administração, Economia e Contabilidade, Universidade de São Paulo.

ZAIRI, M.

O verdadeiro significado da competição.

HSM

Management, ano 1, n. 3, p. 8694, jul./ago. 1997. 